\title{
La evaluación de servicios ambientales de soporte
}

The environmental assessment supporting services

NATALIA SÁNCHEZ-GÓMEZ

Ingeniera Sanitaria y Ambiental

Mg. en Bioética

Grupo de Investigación Gestión Ambiental

Universidad de Boyacá, Colombia

nsanchezg@uniboyaca.edu.co

ZULMA EDELMIRA ROCHA-GIL

Bióloga

Mg. en Ciencias Ambientales

Grupo de Investigación Gestión Ambiental

Universidad de Boyacá, Colombia

zerocha@uniboyaca.edu.co

Recibido: 13/12/2013

Aceptado: 13/02/2014 


\title{
RESUMEN
}

Con la intención de conocer la manera como se ha abordado en la literatura el tema de evaluación de servicios ambientales de soporte, este artículo presenta el resultado de un ejercicio de revisión realizado a través de una lectura crítica. La revisión llevada a cabo, utilizó como criterios de búsqueda las categorías de "Servicios Ambientales" y "Servicios Ecosistémicos", conociendo de antemano que los servicios ambientales de soporte pueden no ser mencionados de manera literal en la producción científica. De los más de 6.700 artículos científicos encontrados sobre el tema, se aplicó un criterio de selección más específico, basado en los autores más citados entre el año 2005 a la fecha, logrando la organización de 57 estudios que permiten brindar un panorama de las diferentes formas como se han evaluado los servicios ambientales de soporte, en experiencias investigativas internacionales y nacionales. El ejercicio realizado permitió evidenciar diferencias conceptuales, limitaciones en cuanto a metodologías de evaluación de servicios ambientales de soporte específicas y vacíos en el estudio de dichos servicios, dejando a la luz la necesidad de profundizar en su estudio en el marco de la sostenibilidad y la resiliencia.

Palabras clave: Servicios Ambientales, Evaluación de Servicios Ecosistémicos, Servicios Ambientales de Soporte.

\begin{abstract}
In order to know how has been addressed in the literature evaluating the issue of environmental support services, this article presents the results of a review exercise conducted through a critical reading. The review conducted used as search criteria categories "Environmental Services" and "ecosystem services", knowing in advance that support ecosystem services cannot be mentioned literally in scientific production. Of the more than 6,700 scientific papers found on the subject, a selection criterion based on the most cited authors from 2005 to date, making the organization of 53 studies to provide an overview of the different forms was performed as have evaluated the environmental support services in national and international research experiences. The exercise made it possible to demonstrate conceptual inconsistencies, limitations on assessment methodologies specific environmental services and gaps in support alternatives to improve such services, leaving to light the need for further study of these services under the sustainability and resilience.
\end{abstract}

Keywords: Environmental Services, Ecosystem Service Assessment, supporting services. 


\section{INTRODUCCIÓN}

La amplia gama de servicios que proporcionan los ecosistemas naturales permite mantener el equilibrio del planeta. En los últimos tiempos, la expansión acelerada de las actividades humanas provoca una profunda modificación de los ambientes naturales, generando múltiples problemas en la conservación de la naturaleza (Costanza et al., 1997, citado por Viglizzo, 2012; Ecosistemas del Milenio Evaluación MEA 2005; PNUMA 2005, citados por Martínez y Gajardo, 2008). Teniendo en cuenta lo anterior y considerando que los servicios del ecosistema son esenciales para la vida en la tierra, así como un indicador útil de la salud del ecosistema (Baker, Sheate, Phillips y Eales, 2013), el concepto de servicios ambientales representa en la actualidad una de las concepciones más utilizadas en el campo de conservación de la biodiversidad, la gestión de los recursos naturales, el desarrollo de políticas, la contabilidad ambiental, entre otros (Jax et al., 2013). Por otro lado, Balvanera (2013), indica que con el paso de los años, en el mundo se está perdiendo la biodiversidad, lo que se traduce en inequidad y pobreza, "cada vez que perdemos una especie, colapsa un servicio ambiental", de ahí la importancia de profundizar, no solo en los estudios, sino también en acciones que contribuyan al buen aprovechamiento de los Servicios Ecosistémicos. Este artículo presenta una revisión general sobre el concepto de servicios ambientales y la manera como se han evaluado los mismos, cuestionando la aplicabilidad de los diferentes métodos y propósitos al evaluar servicios ambientales.

El concepto de Servicios Ecosistémicos - SE, surge del movimiento ambientalista de Estados Unidos en la década de 1970 (Daily, 1997), pero también de la conceptualización creciente de la naturaleza como conjuntos de sistemas integrados (Odum, 1989). Según Laterra, Jobbagy y Paruelo (2010), los Servicios Ecosistémicos - SE y los Servicios Ambientales - SA, son equivalentes sólo de forma parcial. El primero se utiliza en contextos académicos y algunos programas internacionales para enfatizar que los servicios son producto de la interacción entre los distintos componentes de los ecosistemas. El segundo hace referencia a "ambiente" o "medio ambiente" para armonizar con el léxico de secretarías o ministerios en el ramo (SEMARNAT 2003; Balvanera y Cotler 2007). Para fines de este articulo los SE y los SA se mencionarán de manera indistinta.

En este sentido, Jax et al., (2013) han propuesto diversas definiciones, la idea central del concepto de los servicios de los ecosistemas es que contribuyen al bienestar humano. La creación del término 
trasciende la necesidad de conservar la naturaleza y su biodiversidad por sí mismas (Daily, 1997; MEA, 2003). Este enfoque se sugiere como una alternativa para mostrar que la conservación de los ecosistemas no es sólo una aspiración ética de la sociedad, sino también una necesidad estrechamente ligada a la satisfacción de las necesidades básicas de la vida humana (Laterra et al., 2010).

El contenido de este artículo se basa en los hallazgos referentes al tema de evaluación de servicios ambientales de soporte dentro de una revisión de literatura realizada de manera crítica y sistematizada, los resultados se han organizado en tres partes, la primera expone un marco conceptual, intentando resaltar los servicios ambientales de soporte como categoría principal. La segunda parte presenta los diferentes enfoques de evaluación de servicios ambientales de soporte, donde se distinguen tanto las metodologías utilizadas como los diferentes usos y aplicaciones que se han dado a los resultados de la evaluación. La tercera parte resume y organiza la información encontrada, destacando los vacíos y las limitaciones en el tema, con el fin de dar soporte a la generación de las conclusiones, las cuales representan el final del artículo.

El presente ejercicio de revisión se realizó en el marco del macroproyecto "Evaluación de servicios ambientales de soporte en zonas con revegetalización, vereda Ritoque Alto, Municipio de Villa de Leyva”, del Grupo de investigación en Gestión Ambiental de la Universidad de Boyacá y representa la base de los objetivos propuestos en el mismo. Se espera que este documento promueva iniciativas investigativas en el tema de servicios ambientales de soporte.

\section{MARCO CONCEPTUAL DE LOS SERVICIOS AMBIENTALES}

Según Balvanera y Cotler (2007) el concepto de Servicios Ecosistémicos surge de la necesidad de enfatizar la estrecha relación que existe entre los ecosistemas y el bienestar de las poblaciones humanas. Por otro lado, Fisher, Turner, y Morling (2009), relacionan el uso del término "servicios del ecosistema" como un concepto que a partir de George Perkins Marsh, con su trabajo Hombre y Naturaleza en el año 1864, y otros aportes como el de Leopoldo, "ética de la tierra", han generado implicaciones en la ecología experimental y el papel de la biodiversidad en el funcionamiento de los ecosistemas. A pesar de la historia del concepto solo hasta hace poco la literatura se encarga de distinguir exactamente cómo se deben definir los servicios del ecosistema (Boyd, 2007; Barbier, 2007, citados por Fisher, Turner y Morling, 2009).

Según Fisher, Turner, y Morling (2009), existen tres definiciones comúnmente citadas:

- Las condiciones y procesos a través de los cuales los ecosistemas naturales y las especies que los conforman, sostienen y cumplen con la vida humana (Daily, 1997). 
- Los beneficios que poblaciones humanas derivan, directa o indirectamente, de funciones de los ecosistemas (Costanza et al., 1997, citado por Viglizzo, 2012).

- Los beneficios que las personas obtienen de los ecosistemas (MEA, 2005).

De igual manera, Viglizzo, Paruelo, Laterra y Jobbagy (2012) plantean tres conceptos expuestos de la siguiente manera: ( i ) servicios de los ecosistemas son los beneficios que los humanos obtienen de la naturaleza (por ejemplo, alimentos, regulación del clima), ( ii ) las funciones del ecosistema (por ejemplo, el flujo de energía y ciclo de nutrientes que involucra los procesos esenciales que contribuyen a la generación de otros servicios, y ( iii ) los procesos del ecosistema que comprenden la transferencia de energía (por ejemplo, la energía química), material (por ejemplo, alimentos, agua) y la información (por ejemplo, genes, información cultural).

Boyd y Banzhaf (2007) (citados por Fisher, et al., 2009), ofrecen una definición alternativa. En ella, los servicios de los ecosistemas no son los beneficios que los seres humanos obtienen de los ecosistemas, sino los componentes ecológicos directamente consumidos o disfrutados que producen el bienestar humano.

Boyd y Banzhaf (2007) (citados por Fisher, et al., 2009) definen los servicios de los ecosistemas como los aspectos de los ecosistemas utilizados (de manera activa o pasiva) para producir el bienestar humano, por lo cual se puede inferir que los servicios ambientales dependen de la forma como los seres humanos los emplean, indistintamente de la estructura y función de los ecosistemas.

Otra concepción importante la presenta De Groot (1992) (citado por De Groot, et al., 2010), quien afirma que las funciones del ecosistema son intermedias entre los procesos y servicios de los ecosistemas y se pueden definir como la capacidad de los ecosistemas para proporcionar bienes y servicios que satisfagan necesidades humanas, directa e indirectamente. El uso real de un bien o servicio proporciona beneficios (nutrición, salud, placer, entre otros), que a su vez pueden ser valorados en términos económicos y monetarios. Aunque la estructura general de esta cascada es generalmente la distinción entre la función, servicio dado y beneficio, todavía se discute. Balmford et al., (2008) (citado por De Groot, et al., 2010), utiliza los términos proceso de ecosistemas (por ejemplo, producción, descomposición, nutrientes y el ciclo del agua), proceso beneficioso del ecosistema (por ejemplo, producción de biomasa, polinización, control biológico, hábitat y perdida de asimilación) y beneficio (por ejemplo, alimentos, agua dulce, materiales, energía y bienestar) (De Groot, Alkemade, Braat, Hein y Willemen, 2010) (ver Figura 1). 


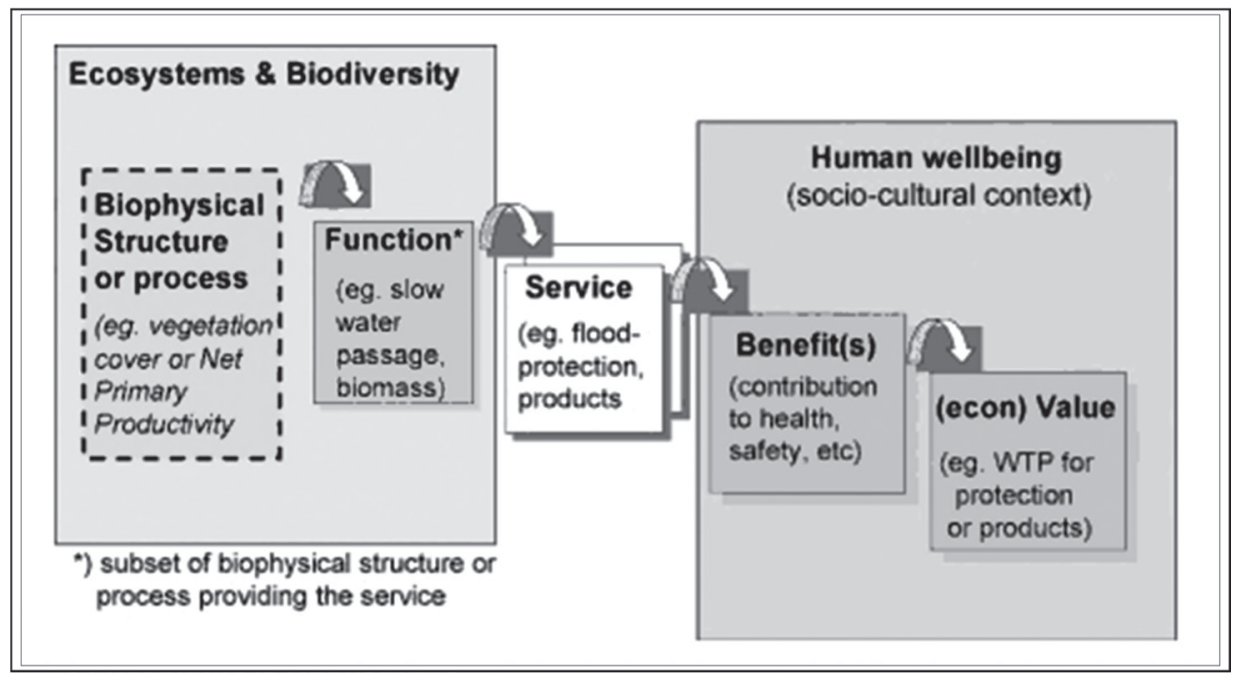

Figura 1. Relación del ecosistema con el bienestar humano. Fuente: De Groot, 2010

En otras definiciones (Reino Unido NEA, 2011, citado por Jax et al., 2013) los componentes y procesos biofísicos que conducen al bienestar humano se llaman "Servicios Ecosistémicos". Otros conceptos (Evaluación de Ecosistemas del Milenio, 2005) establecen que el término se utiliza para identificar los beneficios derivados de los ecosistemas, pero en uno u otro caso, los "servicios del ecosistema" es un concepto cargado de valores y por lo tanto resulta propenso a las controversias sobre los valores específicos que se destacan y los que no se ven (Peterson et al., 2010, citado por Jax et al., 2013).

Otra conceptualización desarrollada a nivel de Latinoamérica son los aportes realizados por Balvanera et al., (2009), quienes mencionan que existen varias formas de clasificar los Servicios Ecosistémicos. La más común los divide en bienes y servicios, para destacar la diferencia entre lo que consumimos, que es tangible, y aquello que nos beneficia de manera menos tangible. Sin embargo, esta clasificación no permite un vínculo explícito entre la forma en que se proporcionan los servicios y la forma en que la sociedad se favorece.

En la revisión, el concepto más citado es el expuesto en el documento de Evaluación de Ecosistemas del Milenio (MEA) de las Naciones Unidas (2005), definiendo los servicios de los ecosistemas como los beneficios que los humanos obtienen de funciones de los ecosistemas y recursos, teniendo en cuenta las diferentes filosofías conceptuales, las cuales se muestran en la Tabla 1. 


\begin{tabular}{|c|c|c|}
\hline Definition of ecosystem services & Citation & Philosophy \\
\hline ..."the benefits human populations derive, directly or indirectly, from ecosystem functions." & (Costanza & Ecosystem ser \\
\hline $\begin{array}{l}\text {..."the conditions and processes through which natural ecosystems, and the species that make them up, } \\
\text { sustain and fulfill human life." }\end{array}$ & (Daily, 199 & nefits \\
\hline $\begin{array}{l}\text {..."the capacity of natural processes and components to provide goods and services that satisfy human needs, } \\
\text { directly or indirectly." }\end{array}$ & (de Groot et al., 2002) & Ecosystem services $\rightarrow$ benefits \\
\hline ..."the set of ecosystem functions that is useful to humans." & (Kremen, 2005) & Ecosystem services $\rightarrow$ benefits \\
\hline ..."the b & (N & Ecos \\
\hline ..."components of nature, directly enjoyed, consumed, or used to yield human well-being." & Banzhaf, 2007) & Ecosystem services $\rightarrow$ benefits \\
\hline ..."the aspects of ecosystems utilized (actively or passively) to produce human well-being." & (Fisher et al., 2009) & Ecosystem services $\rightarrow$ benefits \\
\hline ..."a range of goods and services generated by ecosystems that are important for human well-being." & (Nelson et al., 2009) & Ecosystem services $\rightarrow$ benefits \\
\hline $\begin{array}{l}\text {..."Benefits that humans recognize as obtained from ecosystems that support, directly or indirectly, } \\
\text { their survival and quality of life." }\end{array}$ & (Harrington et al., 2010) & Ecosystem services $=$ benefits \\
\hline ..."a collective term for the goods and services produced by ecosystems that benefit humankind." & (Jenkins et al., 2010) & Ecosystem services $\rightarrow$ benefits \\
\hline
\end{tabular}

Tabla 1. Diferentes definiciones y clasificaciones de SE. Fuente: Ojea, Martin-Ortega y Chiabai, 2012

En cuanto a clasificación, los servicios ambientales también encuentran diferentes concepciones. Según MEA (2005), los servicios de los ecosistemas son clasificados como servicios de aprovisionamiento, servicios de regulación, hábitat o servicios de apoyo y servicios culturales.

Pons Solé (2010) presenta una clasificación similar, donde se encuentran servicios de abastecimiento (productos que se obtienen de los ecosistemas, como la comida, el agua, la madera), de regulación (beneficios que se obtienen de la regulación de los procesos de los ecosistemas, como la regulación del clima, de las inundaciones, de las enfermedades, de los residuos, de regulación de la erosión, de la calidad del agua), culturales (beneficios intangibles que se obtienen de los ecosistemas, como los servicios recreativos, de estética, de turismo, educativos, espirituales) y funcionales (servicios necesarios para la prestación de otros servicios y el sostenimiento del ecosistema, como la formación de suelos, la fotosíntesis, el ciclo de nutrientes).

De Groot, Wilson y Boumans (2002) presentan una clasificación de los servicios según las funciones del ecosistema de la siguiente forma:

1. Funciones de regulación: Este grupo de funciones se refiere a la capacidad de los ecosistemas naturales y semi-naturales para regular los procesos ecológicos esenciales y los sistemas de soporte de vida a través de los ciclos biogeoquímicos y otros procesos de la biósfera. Además de mantener la salud del ecosistema (y la biósfera), estas funciones de regulación ofrecen muchos servicios que tienen beneficios directos e indirectos para los seres humanos, como el aire limpio, el agua y el suelo, y los servicios de control biológico.

2. Funciones de hábitat: Los ecosistemas naturales proporcionan refugio y hábitat de reproducción de plantas y animales silvestres y por lo tanto, contribuyen con la conservación (in situ) de la diversidad biológica y genética y procesos evolutivos. 
3. Las funciones de producción: Fotosíntesis y nutrientes absorbidos por los autótrofos convierten la energía, dióxido de carbono, agua y nutrientes en una amplia variedad de estructuras de hidratos de carbono que luego son utilizados por productores secundarios para crear una variedad aún mayor de la biomasa viva. Esta amplia diversidad de estructuras de carbohidratos proporciona muchos bienes de los ecosistemas para el consumo humano, que van desde alimentos y materias primas a los recursos energéticos y el material genético.

4. Funciones de información: Dado que la mayor parte de la evolución humana se llevó a cabo en el contexto del hábitat salvaje, los ecosistemas naturales proporcionan una función de referencia "esencial" y contribuyen al mantenimiento de la salud de las personas, proporcionando oportunidades para la reflexión, el enriquecimiento espiritual, desarrollo cognitivo, la recreación y la experiencia estética.

Sin embargo, la clasificación de los servicios de la MEA (2005) (citado por Ojea, et al., 2012) ha sido la más ampliamente adoptada, aunque varios autores recientemente han argumentado que la definición y clasificación de los servicios de la MEA pueden no ser las más adecuadas para contribuciones monetarias. En el estudio realizado por Ojea et al. (2012) se expone una tabla resumen de las principales clasificaciones de los Servicios Ecosistémicos que se han utilizado en la literatura reciente, la cual resulta útil en este marco conceptual y se presenta en la Tabla 2.

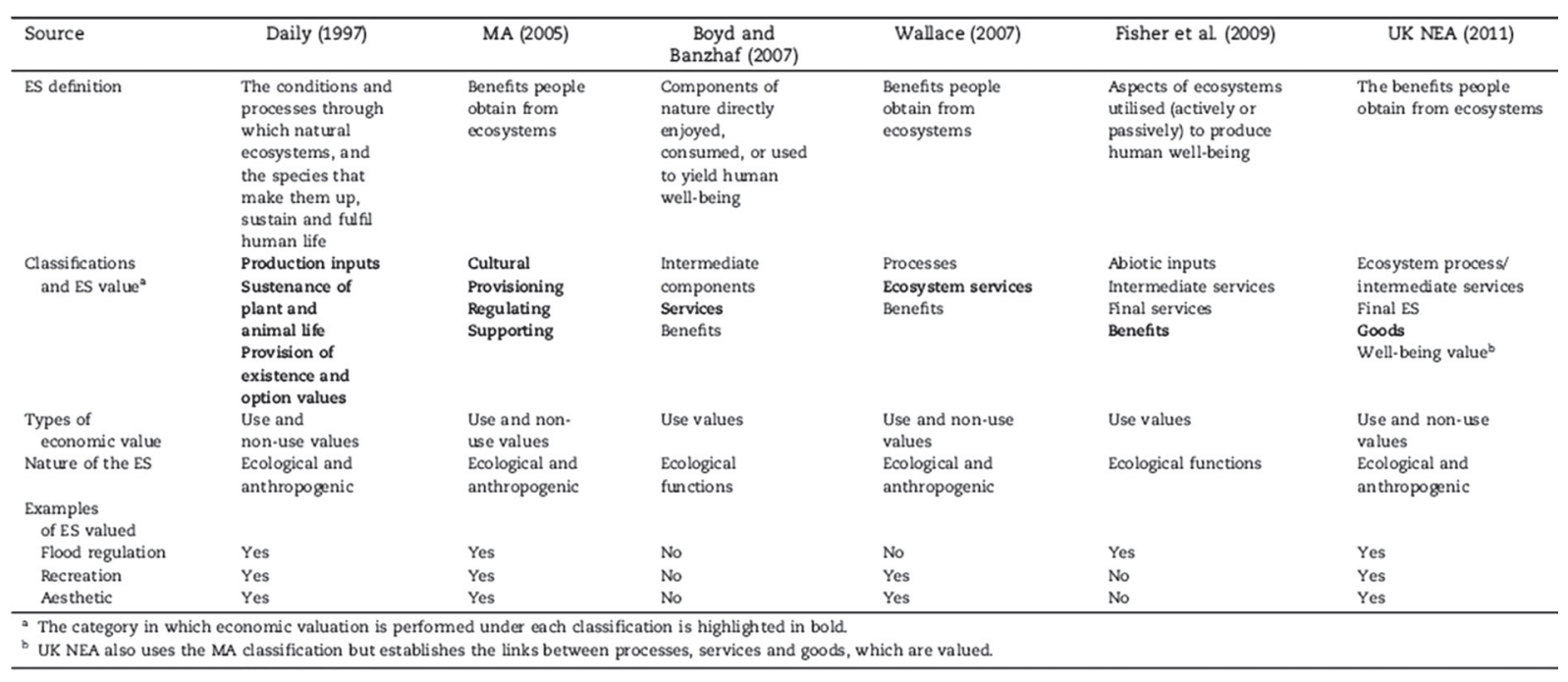

Tabla 2. Diferentes definiciones y clasificaciones de SE. Fuente: Ojea et al., 2012 
En la Tabla 2. Ojea et al., (2012) muestran como algunos autores distinguen entre beneficios y servicios, lo que según ellos, no se debe considerar de la misma forma (Boyd y Banzhaf, 2007; Fisher y Turner, 2008; Bateman, et al., 2011). En su concepto, los servicios son los procesos de los ecosistemas que se relacionan con el bienestar, mientras que los beneficios son los resultados de servicios de los ecosistemas y la relación directa con el bienestar humano (y por lo tanto tienen un significado económico). Basado en esta discusión, algunos autores defienden la valoración de servicios de los ecosistemas por sí mismos (Fisher et al, 2009; Boyd y Banzhaf, 2007), mientras que otros defienden la valoración de ambos servicios de los ecosistemas y los beneficios como elementos separados (Wallace, 2007, citado por Ojea, et al., 2012).

\section{Servicios Ambientales de Soporte}

Analizando las diferentes definiciones y clasificaciones de los Servicios Ecosistémicos o servicios ambientales encontrados en la literatura, emergen algunas diferencias para determinar los servicios ambientales de soporte.

Según la MEA (2005), los servicios de soporte son aquellos que mantienen los procesos de los ecosistemas y permiten provisión del resto de los servicios. Estos pueden no tener implicaciones directas sobre el bienestar humano. Entre ellos se encuentra el mantenimiento de la biodiversidad, el ciclo hidrológico, el ciclo de nutrientes y la producción primaria. De la misma manera, Vonada (2010) conceptualiza a los Servicios de Soporte como Servicios de Apoyo incluyendo el reciclaje y dispersión de nutrientes, la dispersión de semillas además de la producción primaria.

De otra parte, Pons (2010) formula otra denominación al parecer más frecuente en la literatura española, "Servicios Funcionales", definidos como servicios necesarios para la prestación de otros servicios y el sostenimiento del ecosistema, como la formación de suelos, la fotosíntesis y el ciclo de nutrientes. Igualmente Baker (2013) presenta una matriz que permite realizar una evaluación ambiental basada en servicios ambientales, donde incluye como servicios de soporte la formación de suelo, regulación de nutrientes, tratamiento de residuos, polinización, control biológico, provisión de hábitat, función de refugio.

Otros autores, como Viglizzo et al., (2012), asocian a diferentes tipos de servicios ambientales las funciones y procesos ecológicos, como la generación de flujos de energía, ciclos de nutrientes, entre otros (Haygarth y Ritz, 2009, citado por Viglizzo, et al., 2012).

El Tropical Forest Service Flows (2009), clasifica algunos servicios anteriormente mencionados como servicios de soporte dentro de los servicios de regulación, mencionando los siguientes: 
- Regulación de la calidad del aire regional-mundial

- Regulación del clima local-regional-mundial

- Regulación del agua local-regional

- Regulación de la erosión local-regional

- Purificación del agua y tratamiento de residuos local-regional

- Regulación de enfermedades local

- Regulación de plagas local

- Polinización local

- Regulación de peligros naturales local-regional

- La dispersión de semillas local

- La generación de suelo y la fertilidad del suelo local

Power (2010) menciona como servicios de regulación al ciclo de nutrientes la protección del suelo y control de inundaciones, presentándose un traslape entre algunos ejemplos de los servicios considerados como de soporte y los de regulación.

En síntesis, la literatura presenta 4 denominaciones diferentes, al parecer del mismo concepto: servicios ambientales de soporte, de apoyo, funcionales y esenciales, todos fundamentados en que este tipo de servicios generan los demás. Dichas definiciones varían de manera parcial cuando mencionan algunos ejemplos como la dispersión de semillas, el control de la erosión y el ciclo de nutrientes principalmente, las cuales en algunos casos son consideradas como ejemplos de servicios de regulación.

Independientemente de la denominación que se le dé a los servicios ambientales de soporte, lo que queda claro es que así como mencionan Duraiappah et al. (2013), la estrecha interdependencia entre los niveles de la biodiversidad, los procesos y funciones ecológicas, hace que la regulación y el suministro sostenible de aprovisionamiento de los recursos comunes requiera de los servicios de soporte.

Como se puede ver, la importancia de los servicios de soporte se hace evidente en múltiples estudios. Power (2010) señala que el desequilibrio que se produce como respuesta de la pérdida de los servicios esenciales de los ecosistemas es irreversible y que esto aún permanece invisible a los ojos del público. En este sentido, la estimación biofísica de las existencias de biomasa y el agua en los ecosistemas (expresado como valores absolutos o relativos) es una opción ventajosa para, indirectamente, evaluar los flujos asociados con la regulación y servicios culturales prestados por las existencias de biomasa y agua (Power, 2010). 
Según Volante et al. (2012), la conversión de hábitat naturales en tierras de cultivo y tierras de pastoreo en zonas subtropicales, trae consecuencias como la reducción de la actividad fotosintética, así como la alteración en los servicios de los ecosistemas, tales como el control de la erosión y la regulación del agua, debido a la mayor exposición del suelo desnudo. También afecta la biodiversidad debido a la pérdida o disminución de la calidad del hábitat y la disminución de la disponibilidad de biomasa verde para los consumidores primarios durante el barbecho.

Otros estudios incluyen la polinización (considerado como un servicio de soporte), como el mostrado por Tropical Forest Service Flows (2009) donde se relaciona que la polinización forestal será incorporada en los esquemas de Pago por Servicios Ambientales - PSA en el futuro, pues su importancia como un servicio ambiental es incuestionable (Klein et al., 2007), particularmente a la luz de la continua expansión de la superficie cultivada, a expensas de la degradación forestal y/o su conversión (Aizen et al., 2008). En este estudio se sugiere que una forma de asegurar la provisión a largo plazo de los servicios de polinización es a través de la conservación del hábitat (citados por Tropical Forest Service Flows, 2009).

Fisher (2009) menciona los servicios de soporte, afirmando que no son sólo esenciales para la función del ecosistema, sino también un recurso fundamental para el desarrollo sostenible la gestión de los ecosistemas agrícolas. Este estudio está centrado en la biota del suelo donde se muestra su fuerte vínculo con el funcionamiento de los ecosistemas y los servicios ambientales basados en el suelo. Según Fisher (2009), organismos seleccionados desde diferentes grupos funcionales, descomponedores, transformadores elementales, ecosistema del suelo, se utilizan para ilustrar los vínculos de los servicios esenciales para la vida en la tierra, así como con los asociados de la biota del suelo y los ecosistemas con la provisión de bienes y la regulación de procesos ecosistémicos.

Los anteriores son solo algunos ejemplos que confirman la importancia de los servicios ambientales de soporte en el funcionamiento de un ecosistema. Fisher (2009) reconoce la gran diversidad biológica y funcional en el suelo y la complejidad de las interacciones de los sistemas ecológicos, por lo tanto, el suelo es un factor predominante para la optimización de los servicios ambientales de soporte.

\section{EVALUACIÓN DE SERVICIOS AMBIENTALES}

La creciente sensibilidad de la gente respecto a la provisión de bienes y servicios por la naturaleza plantea nuevos desafíos en materia de evaluación de los ecosistemas (Eickhout et al., 2007). Desde mediados de la década de 1980 existe una mayor sensibilización del público acerca de la naturaleza (Stoate et al, 2001; Robinson y Sutherland, 2002), esto ha llevado a reconocer la necesidad de regulaciones, instrumentos y tecnologías para abordar la conservación, desde el punto de vista económico, de servicios de los ecosistemas (Burgess y Morris, 2009, citados por Viglizzo, et al., 2012). 
Viglizzo (2012) manifiesta que la idea de los servicios de los ecosistemas (SE), ha sido ampliamente difundida quizás por su relación con la física valoración del capital (Daily et al., 2009) y porque genera la expectativa para poder contar con métodos operativos para resolver conflictos ambientales y valorar múltiples consecuencias de los cambios de uso.

Dentro de las evaluaciones de servicios ambientales documentadas se encuentra un amplio registro de experiencias en el marco de esquemas de PSA (especialmente en los temas de captura de carbono y regulación hídrica). Balvanera et al. (2012), realizaron una exhaustiva revisión de la literatura de más de 41.000 referencias, encontrando que en América Latina la oferta y la vinculación de Servicios Ecosistémicos a políticas, ha sido la más representativa del mundo, haciéndose énfasis en algunos servicios como carbono y agua . Los pagos por servicios de los ecosistemas han sido objeto de considerable atención en la región, aunque con fuertes diferencias entre las naciones y con limitaciones importantes en su futura aplicación. Sin embargo, según Lindner et al., (2010) (citado por Vesterdal, Clarke, Sigurdsson y Gundersen, 2013) con el creciente énfasis en los servicios de los ecosistemas distintos de la madera procedente de la silvicultura y de creciente interés en la adaptación al cambio climático, hay una necesidad para proporcionar una base más amplia para las decisiones informadas.

Por otro lado, aunque en la mayoría de estudios sobre evaluación de servicios ambientales se presentan en experiencias de PSA, recientes estudios han demostrado que en algunos casos un servicio ambiental basado en el mercado de carbono puede desfavorecer la producción de agua. Power (2010), destaca las relaciones conflictivas que pueden surgir entre la captura de carbono, alcanzado con éxito bajo plantaciones de árboles, y la provisión de agua alcanzada de mejor manera con herbáceas. Jackson et al., (2005) (citado por Power, 2010) menciona que como síntesis de más de 600 observaciones, se pudo documentar que el aumento de carbono a través de plantaciones de árboles causó pérdidas sustanciales en los flujos de corriente, y aumento de la salinización y acidificación. Para Power (2010), esto significa que algunos servicios como la captura de carbono, pueden generar dificultades de otros servicios que afectan a la economía del agua y causar efectos colaterales negativos.

Lo anterior, ha permitido dar una mirada diferente a la prestación de servicios ambientales, dejando de lado el objetivo de evaluar un servicio ecosistémico con fines de "valoración económica", este es el caso de la evaluación de servicios ambientales de soporte, los cuales al no ser considerados como bienes tangibles, no han podido ser incluidos en el marco de PSA, y su estudio se ha desarrollado con miras al desarrollo de políticas de planeación del espacio y usos de suelo, los procesos de toma de decisiones, conservación de la biodiversidad y restauración ecológica.

El problema de la evaluación de servicios ambientales cuando no existen mercados (como en el caso de los servicios de soporte), es la limitación para establecer cuantitativamente los cambios en el uso del suelo, aunque sea bien sabido que estos cambios modifican el nivel de prestación de un determinado servicio ambiental (es decir, las funciones de producción de SE los cuales generan bienes 
y servicios con valor de mercado) (Daily, et al., 2009). Por lo anterior, la evaluación de los servicios ambientales de soporte cobra gran relevancia en la planeación sostenible del espacio más que en la "valoración" de servicios.

En el ámbito de la toma de decisiones, numerosos esfuerzos se están realizando para que el concepto de servicios de los ecosistemas sea funcional y se conecte con planeación. China, México y el Reino Unido llevan a cabo evaluaciones de la biodiversidad y los Servicios Ecosistémicos, abriendo nuevos caminos en el desarrollo de enfoques que funcionan con datos disponibles, la identificación de informes de indicadores y otras formas de comunicar sus hallazgos al público (Bateman et al, 2013;. CONABIO, 2006; Daily et al, 2013; Ehrlich et al, 2012, Reino Unido NEA, 2011, citados por Ruckelshaus, et al., 2013)

Una de las metodologías más presentes en la literatura es el Análisis de Decisiones Multicriterio (MCDA). Según Viglizzo (2012), el modelo MCDA es una herramienta útil para abordar las preguntas del medio ambiente con base en los criterios de casos específicos donde se aplica. Esto, hace que sea difícil comparar los resultados, pero permite combinar la cuantificación ecológica de los servicios de los ecosistemas proporcionados con la importancia asignada de los servicios de los ecosistemas individuales (Fontana, et al., 2013) (ver Figura 2). 


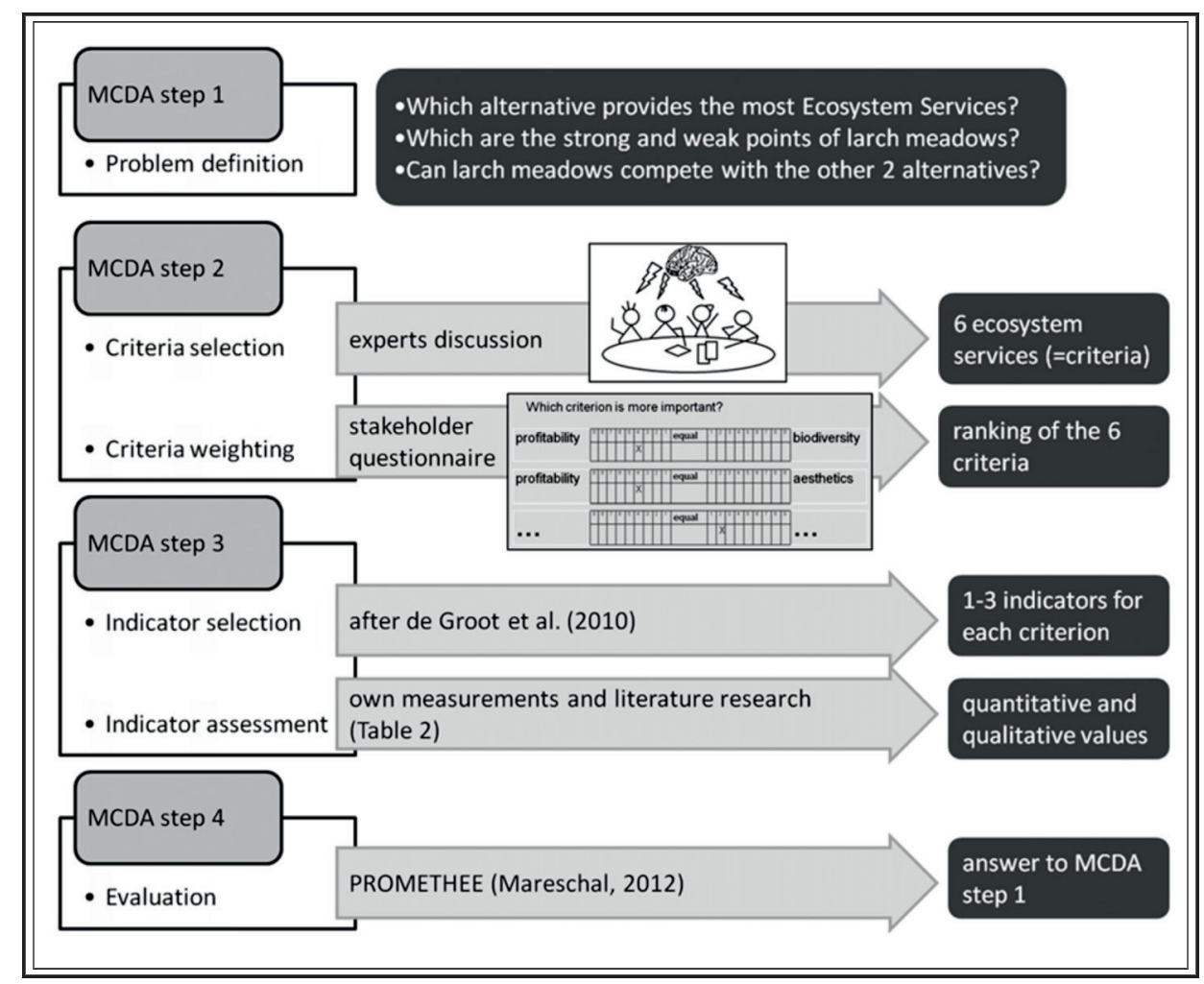

Figura 2. Esquema metodológico de la Evaluación MCDA . Fuente: Fontana, et al., 2013

Fontana et al., (2013), expone un estudio de caso de los Alpes centrales, donde se compararon tres alternativas de uso del suelo en diferentes condiciones: el alerce (pradera tradicional), bosque de abetos (abandono) y la pradera intensiva (intensificación). Los criterios para el modelo MCDA fueron seleccionados por expertos, los criterios de importancia fueron clasificados por los interesados y los valores de los criterios fueron evaluados con indicadores cualitativos y cuantitativos. Eventualmente el bosque de abeto fue clasificado como la mejor alternativa de uso del suelo, seguido de prado alerce tradicional y prado intensivo. El enfoque combinado de MCDA mostró cómo los criterios y valores ponderados de los indicadores para definir servicios ambientales influyen en el rendimiento de las alternativas de uso del suelo. Este estudio concluye que el modelo MCDA visualiza las consecuencias de cambio de uso de la tierra para la prestación de servicios de los ecosistemas, lo que facilita la planificación del paisaje mediante la estructuración de los problemas ambientales, proporcionando datos para las decisiones. 
En política de uso de la tierra existe una creciente preocupación sobre la forma de incorporar igualmente, los indicadores sociales y económicos y las valoraciones de los ecosistemas de servicio, en una matriz de toma de decisiones bien equilibrada (Kremen, et al., 2005). Las metodologías de evaluación de servicios ambientales con enfoque de uso de suelo son variadas pero semejantes en cuanto al uso de estudios comparativos y evaluaciones in situ.

Raudsepp - HearMe et al., (2010) (citados por Power, 2010) desarrollaron un marco llamado "Conjunto de análisis del servicio del ecosistema" para el análisis de la provisión de múltiples servicios de los ecosistemas a través de paisajes. En el caso de Argentina y sobre la base de estimaciones, se desarrolló un conjunto de análisis de ventajas y desventajas entre el estimado margen bruto anual por hectárea y el valor de servicios de los ecosistemas en tres periodos históricos. Allí se muestra que los beneficios de los servicios económicos aumentan exponencialmente a medida que los Servicios Ecosistémicos declinan linealmente en el tiempo y el espacio. En términos de recursos humanos se percibe que la provisión de beneficios económicos tangibles obtuvo abrumadoras pérdidas de los servicios ecológicos intangibles. Así es como se señala que este desequilibrio se produce a expensas de los servicios esenciales de los ecosistemas.

Viglizzo (2012) presenta otro método empleado en la evaluación de Servicios Ecosistémicos: la evaluación Biofísica de biomasa y agua y sus flujos asociados en ecosistemas terrestre. Con este método se han realizado las evaluaciones en las diferentes áreas de Argentina, como las Pampas del Sur (Laterra et al, 2012), los de alta productividad de los Rolling Pampas (Caride et al, 2012; Gavier - Pizarro et al, 2012), la región de transición entre las pampas occidentales y la región de Espinal (Nosetto et al., 2012) y la transición entre el subtropical húmedo bosques (Yungas) y los bosques secos subtropicales (Chaco) en el noroeste de Argentina (Volante et al., 2012). Todos estos estudios asumieron que algunas funciones ecológicas de la vegetación y el agua en el paisaje pueden estar asociadas a un número de servicios de agregados como secuestro de carbono, protección del hábitat y la vivienda.

En esta misma dirección, Marinidou et al., (2012), presentan un método adaptado de ecología funcional para evaluar servicios ambientales de mantenimiento de biodiversidad, donde se estima el aporte de la cobertura arbórea a la conservación de especies. Esta contribución la definieron en términos de tres "sub- servicios": alimento y hábitat de provisión para la fauna silvestre, y la existencia o el valor intrínseco de las especies arbóreas. El valor de existencia es un concepto difícil de medir y es fuertemente dependiente culturalmente (Groom et al., 2006), pero se comparte la opinión de Naess (1986) de que el valor de conservación no sólo debe ser definido en términos utilitarios. Esta metodología propone pasar de los rasgos de las especies de árbol de tipo de cobertura y cultivar valor ecológico en tres pasos (cf. Díaz et al., 2007). En primer lugar, se propone un índice de valor funcional de especies basándose en las características ecológicas y los rasgos funcionales, luego se calcula el valor ecológico de cada árbol que combina las especies de valor funcional y dimensiones individuales de los árboles. 
Esta metodología se fundamenta en determinar el valor funcional para cada servicio ambiental basado en especies arbóreas, caracterizando las especies de árboles en cuanto a su potencial para generar la regulación del clima, conservación de la biodiversidad y servicios con la combinación de los valores medios de características como: valor de los alimentos para la fauna silvestre, el valor del hábitat para la fauna silvestre, valor de existencia, provisión de frutas, semillas, provisión de conectividad, recursos de forraje / follaje y florales (Thiollay, 1995) supervivencia de la fauna silvestre en estaciones secas (Foster , 1990), su importancia general para los vertebrados (Faller - Menéndez et al. , 2005), y la influencia de la corona de las especies de árboles y el parche. Los árboles, además de proporcionar alimento y hábitat para otras especies, también agregan valor a la biodiversidad de un paisaje únicamente por estar allí. Para ello, se refiere al valor de existencia, como uno de los tres componentes para evaluar el valor de árboles por su contribución a la conservación de la biodiversidad (Marinidou, et al., 2012).

Marinidou et al., (2012) afirman que las especies de árboles de valor ecológico funcional indican su posible contribución a un servicio ambiental, útil como un criterio para la selección de especies para promover un servicio requerido. Sin embargo, los servicios ambientales difieren también con respecto a las dimensiones y características de la comunidad quien influye en su potencial. Este estudio realizó una evaluación ecológica de 140 especies de árboles y siguiendo este método es sencillo estimar los valores para muchas más especies.

La evaluación de los servicios de los ecosistemas todavía muestra una serie de diferencias en el enfoque y los métodos para determinar valor de los servicios ambientales relacionados con el uso del suelo (Wunder et al., 2008). Tales diferencias pueden reflejar, entre otras cosas, las brechas socio-económicas y culturales entre las comunidades, cierta subestimación de la naturaleza como proveedora de servicios de los ecosistemas, y desacuerdos acerca de los métodos de valoración (Kosoy et al, 2007; Engel et al., 2008, citados por Kosoy, Martínez-Tuna, Muradian y Martínez-Alier, 2007).

Según Fisher (2009), existen enfoques metodológicos, experimentales y conceptuales útiles para el reto de vincular la biodiversidad y la función del suelo con la prestación de servicios de los ecosistemas. Estos incluyen investigación sobre variabilidad espacial del suelo y la integración con la actividad biológica, el uso de grupos funcionales en la biota del suelo, la dinámica temporal y espacial de organismos, y su contribución a la prestación de Servicios Ecosistémicos mediante la comprensión de: las relaciones jerárquicas de la biota y la función del suelo en los sistemas de cultivo, el conocimiento local sobre las plantas como indicadores de la calidad del suelo, las tecnologías de detección a distancia GIS, las interacciones de la biota planta-suelo a escala de paisaje, el desarrollo de sistemas de monitoreo de la calidad del suelo, que informan a los usuarios de la tierra sobre el desempeño de Servicios Ecosistémicos de la tierra, mejorando la capacidad de predecir su funcionamiento. Fisher (2009) afirma que mediante la comprensión de las características clave estaremos más preparados para gestionar, mantener, restaurar o evaluar servicios de los ecosistemas. 
Por otro lado, Baker et al., (2013) presentan una metodología de evaluación que toma un enfoque de estudio de caso empírico para explorar cómo los servicios de los ecosistemas se han utilizado en Estudios de Impacto Ambiental y Evaluaciones Ambientales Estratégicas. Este estudio ilustra ejemplos de aplicación práctica de los servicios de los ecosistemas en la evaluación ambiental. Allí se han seleccionado los estudios de caso para ilustrar una serie de cuestiones que surgen de la actual práctica ambiental de la evaluación y cómo un enfoque basado en los servicios de los ecosistemas puede o no puede ayudar a abordar estas cuestiones. El documento concluye con un análisis de la relación, los pros y los contras de la aplicación del concepto de Servicios Ecosistémicos en la evaluación del medio ambiente con referencia a los temas y principios generales. Presenta igualmente una matriz que permite realizar una evaluación ambiental, incluyendo como servicios de soporte la formación de suelo, regulación de nutrientes, tratamiento de residuos, polinización, control biológico, provisión de hábitat, función de refugio (Baker, et al., 2013).

Es importante mencionar que gran parte de la literatura encontrada refería el término de "valoración" y no "evaluación" de Servicios Ecosistémicos, la mayoría de experiencias de evaluación se han realizado con fines de establecer mercados, así lo afirma Viglizzo (2012): "el precio es uno de los indicadores más frecuentes utilizados en los estudios de servicios ambientales".

Carpenter et al., (2009) (citado por Viglizzo 2012) muestra cómo el costo de proyectos de ingeniería para el tratamiento de la regulación del flujo de agua se han utilizado para estimar indirectamente el valor de mercado de los Servicios Ecosistémicos. Del mismo modo, el costo económico alrededor de la recreación y el turismo (viajes, alojamiento, y otros bienes y servicios) se utiliza como una herramienta para estimar el valor económico de los servicios culturales que contribuyen al bienestar humano.

Así mismo el escrito de Costanza et al. (1997) (citado por Viglizzo, 2012), el cual constituye probablemente el análisis más completo y conocido en este tema, demuestra con base en el concepto de utilidad para los seres humanos, que "sin valor" el uso de algunos activos naturales, como la diversidad de especies, es totalmente ignorado, dejando de lado el valor de la existencia de especies, la cual contribuye a proveer los servicios esenciales que son de interés humano.

Esta valoración económica de los servicios ambientales ha sido criticada como un mecanismo para la toma de decisiones cuando la información y los datos son el resultado de estimaciones subjetivas, entre las diferentes categorías de consumidores (Carpenter, et al., 2009). De esta manera, la valoración económica se mantiene como uno de los aspectos más estudiados de los servicios ambientales y la falta de una valoración monetaria adecuada se identifica como un motor importante de la disminución de la provisión de SA (MEA, 2005). A pesar del esfuerzo aplicado para desarrollar prácticas efectivas y métodos de valoración de Servicios Ecosistémicos para la formulación de políticas, los ejemplos exitosos son relativamente raros (Ruffo y Kareiva, 2009, citados por Viglizzo 2012), también 
se evidencia desarrollo del tema sectorizado, como el estudio realizado por Khare y White (2003) sobre mercados para Servicios Ecosistémicos de bosques tropicales, en el cual se describe el interés de estos mercados, formas de pago, beneficios y riesgos, entre otros, pero además concluye que los conocimientos y experiencias en el tema, se orientan a mercados en el sector privado, generalmente en compañías y consultores motivados por la oportunidad de promover negocios comerciales.

Teniendo en cuenta todo lo anterior, se cuestiona si este enfoque económico reúne todos los criterios o aspectos para realizar un uso sostenible y resiliente de los ecosistemas o, como afirma Ludwig (2000), el valor económico no captura adecuadamente el valor intangible de servicios ambientales de regulación y culturales que no pueden ser fácilmente cuantificados en términos de precio. Además, la valoración económica según Layke (2009) sólo tiene en cuenta los indicadores del flujo de un servicio ambiental (alimentos, fibras, energía, materias primas) en lugar de la acción de los bienes naturales que miden la capacidad del ecosistema para prestar un servicio.

De igual manera en la última década, los esfuerzos para valorar y proteger los servicios de los ecosistemas han sido promovidos por muchos como la mejor esperanza para la conservación en todo el mundo (Daily, et al., 2009). En teoría, según Daily et al., (2009), si se ayuda a las personas e instituciones a reconocer el valor de la naturaleza, aumentarán considerablemente las inversiones en la conservación, mientras que al mismo tiempo se fomenta el bienestar humano. En la práctica, sin embargo, todavía no está desarrollada la base científica, ni los mecanismos de política y finanzas, para la incorporación de capital natural en recursos y las decisiones de uso de tierras a gran escala.

La valoración económica de los servicios ambientales y los posteriores beneficios, es fundamental para la gestión sostenible de los ecosistemas. Dado que estos servicios devengados a la sociedad son públicos en la naturaleza y considerados intangibles, suponen un reto formidable en la valoración. Algunos de los retos de la valoración emergen del sesgo de la economía neoclásica, por esto Kumar y Kumar (2008), muestran un ejercicio de valoración basado en la necesidad el concepto de identidad ecológica, la interdisciplinariedad y el pluralismo metodológico.

Centrándose en Colombia, las experiencias documentadas se limitan a metodologías de evaluación implementadas para la valoración de servicios ambientales con miras a la implementación de esquemas de PSA. Una de las experiencias más significativas se presenta en Ruckelshaus (2013) quien expone un nuevo mecanismo para la protección de los servicios de las cuencas hidrográficas que surgen en América Latina, en la región de los Andes, donde una mezcla de los humedales de gran altitud (páramo) y bosques proporcionan una valiosa regulación del suministro de agua y un aporte al control de la erosión y a la retención de nutrientes, que mejora la calidad del agua a los millones de personas en las montañas y los valles andinos. 
Según Ruckelshaus (2013), con este conocimiento se están desarrollando esquemas que permiten el pago a los administradores de tierras aguas arriba para mejorar la gestión de cuencas hidrográficas como una forma de regular los flujos de agua y proporcionar filtración natural para la calidad del agua. Estos pagos por servicios de cuencas son creados por un grupo de usuarios que pagan y luego colectivamente deciden cómo invertir en la gestión de cuencas (Goldman- Benner et al., 2012). Uno de estos fondos para el agua, agua para la vida y la sostenibilidad, ha sido creado en Cali, Colombia con el apoyo de cada una de las cuencas hidrográficas locales, la autoridad ambiental, la asociación de productores de caña de azúcar (ASOCAÑA), la asociación de cultivadores de caña de azúcar (ProCaña), el Nature Conservancy, y Vallenpaz (organización de la paz y la justicia). Su objetivo es mantener consistentes flujos de agua necesarios para beber, la biodiversidad y la agricultura a través de una coordinada estrategia (citados por Ruckelshaus, 2013).

En el país, el tema de evaluación de servicios ambientales ha sido abordado en espacios académicos y científicos como en el III Congreso Internacional de Servicios Ecosistémicos realizado en el año 2013 en la Universidad Nacional de Medellín, en donde se han destacado avances importantes, como el reconocimiento del concepto de Servicios Ecosistémicos en las personas y la creación de redes de trabajo en pro de la conservación de los ecosistemas naturales que hay en la región. Además, Colombia cuenta con una Política Nacional para la Gestión Integral de la Biodiversidad y sus Servicios Ecosistémicos, al igual que normatividad reglamentaria expuesta en el Decreto 0953 de 2013 en el cual se establecen los Pagos por Servicios Ambientales, con el fin de promover la conservación y recuperación de las áreas de importancia estratégica para la conservación de recursos hídricos que surten de agua a los acueductos municipales, distritales y regionales, mediante la adquisición y mantenimiento de dichas áreas y la financiación de los esquemas de pago.

Según Villegas (2012), a nivel nacional se han desarrollado trabajos en el Departamento de Antioquia para la valoración económica, ecológica y socio-cultural de bienes y servicios ambientales y se han identificado áreas importantes para la provisión de estos, considerando como principales servicios ambientales los de aprovisionamiento, regulación, soporte, hábitat y culturales.

Según Ruíz et al., (2007) en la revisión de estudios de valoración económica de los andes colombianos, se recopilaron 100 contribuciones (principalmente trabajos de pregrado y documentos técnicos de ONGs), sobre la valoración económica de Servicios Ecosistémicos, determinando que el conocimiento generado sobre valoración en ecosistemas alto andinos a nivel nacional es aceptable, teniendo en cuenta que la economía ambiental como disciplina no es tan reciente, pero sí lo es como herramienta en los procesos de gestión ambiental. También definió que los ecosistemas andinos han sido valorados en términos de la conservación del recurso hídrico, con el fin de asegurar la oferta de agua en cantidades y calidades adecuadas, reconociendo su importancia entre los principales Servicios Ecosistémicos que ofrecen los andes colombianos, definiendo acciones asociadas a la conservación de la biodiversidad para a través de estas, buscar asegurar la provisión de Servicios Ecosistémicos de soporte. 
De la misma manera Ruíz et al., (2007) afirma que la mayoría de estudios en esta región se relacionan con la regulación de ciclo hidrológico, debido probablemente a que esta es la función más importante que se reconoce de estos sistemas, aunque también se han realizado algunos estudios relacionados con la perspectiva de sumideros de carbono, sin embargo este servicio ha sido poco caracterizado a la luz de los estudios existentes. Se reportaron varios estudios en los departamentos de Antioquia y Santander, valorando el recurso hídrico; no obstante zonas como Boyacá, Cauca y Norte de Santander carecen de este tipo de estudios. En el departamento de Cundinamarca se reportan estudios de valoración económica correspondientes a ecosistemas de humedales, específicamente en Bogotá, en donde se valoraron los Humedales de Córdoba, La Conejera, La Florida, y Juan Amarillo y estudios en las áreas protegidas de la región Andina, de éstas solo se conocen trabajos en el Santuario de Flora y Fauna de Iguaque, y los Parques Nacionales de Chingaza y Los Nevados.

En el proceso de valorar los Servicios Ecosistémicos de los Andes colombianos, se han adelantado varias iniciativas para estimar el valor que la sociedad otorga a los bienes y servicios derivados de los ecosistemas, implementando entre otros los siguientes métodos: valoración contingente Bullón, (1996); Arango, (1999); Pinzón, (1999); Camelo, (2008) valoración conjunta Pérez y Aguirre, (2000), costos de viaje Bullón, (1996); Rodríguez de Francisco, (2003), costos de oportunidad Castillo, (2008), entre otras (citados por Ruíz et al., 2011). Sin embargo, estos son estudios puntuales que abarcan parte de los ecosistemas de los Andes, ya que Buitrago y Ruiz (2010) (citados, por Ruíz et al., 2011), en la misma iniciativa, revelan que aún quedan muchas zonas importantes por valorar en los departamentos como Cauca, Popayán, Risaralda y Huila, entre otros.

\section{VACIOS Y LIMITACIONES EN LA EVALUACIÓN DE SERVICIOS AMBIENTALES DE SOPORTE}

Rodríguez et al., (2011) afirman que las complejas interacciones entre los ecosistemas en peligro de extinción, los intereses de los terratenientes y los diferentes modelos de la tenencia de la tierra y su uso, constituyen una importante serie de retos para los que buscan mantener y restaurar la biodiversidad y aumentar el flujo de servicios de los ecosistemas. En este sentido, como cita Martín y Montes (s.f.) los ecosistemas y la biodiversidad que éstos albergan proveen la plataforma básica para mantener las funciones de los ecosistemas y se cuestiona ¿cuánta y cómo es la estructura esencial para proveer al ser humano de los eco-servicios necesarios para el mantenimiento de su bienestar? Esta es una pregunta que todavía se mantiene abierta y requiere de mayor esfuerzo científico (Kremen, 2005).

De la misma manera, la Comisión Europea sobre la incorporación de la Biodiversidad y el cambio climático en el mar y procedimientos de EIA (CCA, en prensa) sugiere que el concepto de servicios de los ecosistemas es útil para considerar cuestiones tales como el cambio climático y la adaptación y la resiliencia en el medio ambiente (Baker, et al., 2013). Sumado a esto el valor económico total por sí solo

La evaluación de servicios ambientales de soporte 
no puede dar una indicación de la capacidad de un ecosistema para mantener la futura prestación de servicios (Fisher et al., 2008). Admiraal, Wossink, De Groot y Snoo (2013) describen un ejemplo de gestión de un sistema agrícola en el sureste de Australia en el que el mantener la vegetación fue una estrategia para proteger el ecosistema contra inundaciones, lo que permite evidenciar la necesidad de ampliar los horizontes de evaluación.

Además, la toma de decisiones basada en la mejora de la eficiencia económica y la productividad se hace cada vez más reactiva e incremental, conduciendo a la pérdida de la capacidad de recuperación y al manejo no sustentable del ecosistema. Admiraal et al., (2013) explican por qué el valor económico total de por sí es en principio, un concepto insuficiente para guiar el uso sostenible de los ecosistemas, por eso este autor plantea una combinación de puntos de vista desde la teoría ecológica acerca de la resiliencia del ecosistema y una perspectiva económica de la inversión en la biodiversidad. La teoría de este portafolio pone el valor económico en un marco donde la inversión en la biodiversidad se amplía para abarcar la diversidad funcional y el enlace de especie móviles con el fin de mantener la resiliencia del ecosistema y así fomenta el uso sostenible de los ecosistemas (Admiraal, et al., 2013).

Todo lo anterior refleja la dificultad de analizar servicios ambientales de soporte, funcionales, esenciales o de apoyo, dado el interés persistente de los estudios a un enfoque económico y de monetización hacia los bienes y servicios que satisfacen las necesidades humanas, dando menor espacio o importancia a la valoración económica de servicios como el ciclaje de nutrientes, la productividad primaria, la formación de los suelos, entre otros, considerados según el estudio del MEA (2005) como la base para la generación de los demás servicios ambientales, conllevando al deterioro o abandono de la protección y valoración económica de bienes o servicios intangibles y en consecuencia subvalorando la estructura y función ecosistémica de sistemas conservados o intervenidos, que potencialmente representan esta oferta, como por ejemplo los sistemas de bosque secundario o intervenido que son usados o manejados para pastoreo, minería, aprovechamiento forestal, fibras, alimento, captura de carbono, adaptación al cambio climático, entre otros, sin estimar los costos de oportunidad o de valoración económica de servicios de soporte con miras a un verdadero desarrollo sostenible basado en la resiliencia ecosistémica.

\section{CONCLUSIONES}

Las diversas formas de abordar el tema de servicios ambientales de soporte permiten concluir una presencia transversal de este concepto en la literatura, esto demuestra un avance en el reconocimiento de dichos servicios y resalta la importancia de construir conocimiento en este sentido. Sin embargo, el concepto de "servicios de soporte" no se relaciona como categoría de análisis central de estudio, sino como un elemento generalizado o inmerso en estudios de suelos con enfoque de ordenamiento territorial. 
Los servicios ambientales de soporte no representan en sí mismo un objeto de estudio, lo cual impide profundizar en el tema y obtener datos específicos sobre evaluación o análisis de dichos servicios en las investigaciones. En virtud de lo anterior, es importante resaltar la necesidad de construir conocimiento puntual sobre este tipo de servicios para lograr abordarlos de manera más amplia y concreta.

Existe una amplia producción científica y académica reciente en el tema de evaluación de servicios ambientales o ecosistémicos, sin embargo la mayoría de la información revisada presenta casos con escalas incompatibles de análisis y diferente clasificación de servicios ambientales, limitando la trazabilidad del conocimiento y dificultando procesos de análisis comparativo. Se podría afirmar que las experiencias de evaluación surgen, a partir de casos puntuales asociados a los fines de estudio. Además, existen diferencias estructurales en los enfoques y métodos de evaluación de servicios ambientales, demostrados en los diversos esquemas de pago por los mismos, los cuales han incorporado diferentes propósitos y conceptos ecológicos para estimar valor o precio pagado por tales servicios, que muestran heterogeneidad en la aplicación de conceptos e instrumentos de análisis.

Se evidencia en todo el recorrido por los métodos de valoración de servicios ambientales, la necesidad de superar las limitaciones de la visión monetaria de evaluación, a través de enfoques sistemáticos para evaluar la naturaleza, como los propuestos por Odum (2000) o Kremen y Ostfeld (2005) quienes incorporan criterios biológicos y/o sociales que permiten ampliar el espectro de estudio de los Servicios Ecosistémicos hacia la sostenibilidad.

No se presenta un enfoque central en la literatura científica revisada en métodos de evaluación que consideren todos los elementos estructurales y funcionales de los ecosistemas para determinar un servicio específico óptimo, por el contrario, en la mayoría de experiencias documentadas en esta revisión, la evaluación es orientada hacia servicios predeterminados que en la mayoría de los casos son servicios tangibles reconocidos en el ámbito económico, subvalorando o desconociendo otros servicios intangibles que no representan un bien directo para el ser humano, pero que a largo plazo podrían garantizar el mantenimiento de los demás Servicios Ecosistémicos. 


\section{REFERENCIAS BIBLIOGRÁFICAS}

Admiraal, J. F., Wossink, A., De Groot, W. T. de y Snoo, G. R. de. (2013). More than total economic value: How to combine economic valuation of biodiversity with ecological resilience. Ecological Economics, (89), 115-122.

Baker, J., Sheate, W.R., Phillips, P. y Eales, R. (2013). Ecosystem services in environmental assessment — Help or hindrance? Environmental Impact Assessment Review, (40), 3-13.

Balvanera, P. y Cotler, H. (2007). Acercamientos al estudio de los servicio ecosistémicos. Gaceta Ecológica, (84-85), 8-15.

Balvanera, P., Cotler, H., Aburto Oropeza, O., Aguilar Contreras, A., Aguilera Peña, M., Aluja, M., Zarco, A. (2009). Estado y tendencia de los Servicios Ecosistémicos. Capital Natural de México. México. p. $185-245$.

Balvanera, P., Uriarte, M., Almeida-Leñero, L., Altesor, A., DeClerck, F., Gardner, T., Vallejos, M. (2012). Ecosystem services research in Latin America: The state of the art. Ecosystem Services, (2), 56-70.

Balvanera, P. (2013). Evaluación de funciones y Servicios Ecosistémicos, vulnerabilidad y riesgo. Tercer Congreso Internacional de los Servicios Ecosistémicos en los Neotrópicos. Medellín, Colombia: Universidad Nacional.

Brown, G. (2013). The relationship between social values for ecosystem services and global land cover: An empirical analysis. Ecosystem Services, (5), e58-e68.

Daily, G. (ed.). (1997). Introduction: What are ecosystem services. Island Press, Washington, D.C.

Daily, G. C., Polasky, S., Goldstein, J., Kareiva, P. M., Mooney, H. A., Pejchar, L., Shallenberger, R. (2009). Ecosystem services in decision making: time to deliver. Front Ecology Environmental, 7(1), 21-28.

De Groot, R. S., Alkemade, R., Braat, L., Hein, L. y Willemen, L. (2010). Challenges in integrating the concept of ecosystem services and values in landscape planning, management and decision making. Ecological Complexity, (7), 260-272.

De Groot, R. S., Wilson, M. A. y Boumans, R. M. J. (2002). A typology for the classification, description and valuation of ecosystem functions, goods and services. Ecological Economics, (41), 393-408. 
Duraiappah, A. K., Asah, S. T., Brondizio, E. S., Kosoy, N., O'Farrell, P. J. Prieur-Richard A-H, Takeuchi, K. (2013). Managing the Mismatches to Provide Ecosystem Services for Human Well-being: A Conceptual framework for understanding the New Commons. Current Opinion in Environmental Sustainability, doi: http://dx.doi.org/doi:10.1016/j.cosust.2013.11.031

Fisher, B., Turner, K., Zylstra, M., Brouwer, R., De De Groot, R., Farber, S., Ferraro, P., Green,R., Hadley, D., Harlow, J., Jefferiss, P., Kirkby, C., Morling, P., Mowatt, S., Naidoo, R.,Paavola, J., Strassburg, B., Yu, D., Balmford, A., (2008). Ecosystem services and economic theory: integration for policy-relevant research. Ecological Applications 18, 2050-2067.

Fisher, B., Turner, R. K. y Morling, P. (2009). Defining and classifying ecosystem services for decision making. Science Direct, (68), 643-653.

Fontana, V., Radtke, A., Bossi Fedrigotti, V., Tappeiner, U., Tasser, E., Zerbe, S. y Buchholz, T. (2013). Comparing land-use alternatives: Using the ecosystem services concept to define a multi-criteria decision analysis. Ecological Economics, (93), 128-136.

Jax, K., Barton, D. N., Chan, K. N. A., De Groot, R. de, Doyle, U., Eser, U, Wichmann, S. (2013). Ecosystem services and ethics. Ecological Economics, (93), 260-268.

Khare, A. y White, A. (2003). Estado actual y potencial futuro de los mercados para los Servicios Ecosistémicos de los bosques tropicales: panorama general. Consejo Internacional de las Maderas Tropicales. Yokohama, Japón: El Consejo.

Kosoy, N., Martínez-Tuna, M., Muradian, R. y Martinez-Alier, J. (2007). Payments for environmental services in watersheds: Insights from a comparative study of three cases in Central America. Ecologicale Economics, (61). 446-455.

Kremen, C. y R. Ostfeld. (2005). A call to ecologists: measuring, analyzing, and managing ecosystem services (Review). Frontiers in Ecology and the Environment 3:540-548.

Kumar, M. y Kumar, P. (2008). Valuation of the ecosystem services: A psycho-cultural perspective. Science Direct, (64), 808-819.

Laterra, P., Jobbagy, E. G. y Paruelo, J. M. (2010) (Ed.). (s.f.). Valoración de Servicios Ecosistémicos: conceptos, herramientas y aplicaciones para el ordenamiento territorial. Instituto Nacional de Tecnología Agropecuaria.

Layke, C. (2009). Measuring Nature's Benefits: a Preliminary Roadmap for Improving.

Ludwig, D. (2000). Limitations of economic valuation of ecosystems. Ecosystems 3, 31-35. 
Marinidou, E., Finegan, B., Jiménez-Ferrer, G., Delgado, D. y Casanoves, F. (2013). Concepts and a methodology for evaluating environmental services from trees of small farms in Chiapas, México. Journal of Environmental Management, (114), 115-124.

Martínez-Harms, J. M. y Gajardo, R. (2008). Ecosystem value in the Western Patagonia protected areas. Journal for Nature Conservation, (16), 72-87.

Martín-López, B. y Montes, C. (s.f.). Funciones y servicios de los ecosistemas: una herramienta para la gestión de los espacios naturales. Guía científica de Urdaibai. UNESCO, Dirección de Biodiversidad y Participación Ambiental del GobiernoVasco.

MEA. 2005. Millenium Ecosystem Assessment, Ecosystems and human well-being: biodiversity synthesis. Washington, D.C. EE.UU.Nahlik, A. M., Kentula, M. E., Fennessy, M, S. y Landers, D. H. (2012). Where is the consensus? A proposed foundation for moving ecosystem service concepts into practice. Ecological Economics, (77). 27-35.

Odum, E.P. (1989). Ecology and our endangered life support system. Sinauer Associates, Inc.Publishers, Sunderland, Massachusetts. EE.UU.

Odum, H.T. y E.P. Odum. (2000). The energetic basis for valuation of ecosystem services. Ecosystems 3:21-23.

Ojea, E., Martin-Ortega, J. y Chiabai, A. (2012). Defining and classifying ecosystem services for economic valuation: the case of forest water services. Environmental Science and Policy, (19-20), 1-15.

Pons Solé, J. (2010). La evaluación de los servicios ambientales de los ecosistemas litorales en Catalunya. Foro de Sostenibilidad. 65-78.

Power, A.G. (2010). Ecosystem services and agriculture: tradeoffs and synergies (Review). Philosophical Transactions of the Royal Society B 365, 2959-2971.

Rodríguez, R. R., Gandolfi, S., Nave, A. G., Aronson, J., Barreto, T. E., Vidal, C. Y., Brancalion, P. H. S. (2011). Large-scale ecological restoration of high-diversity tropical forests in SE Brazil. Forest Ecology and Management, (261), 1605-1613.

Ruckelshaus, M., McKenzie, E. Tallis, H., Guerry, A., Daily, G., Kareiva, P. Bernhardt, J. (2013). Notes from the field: Lessons learned from using ecosystem service approaches to inform real-world decisions. Ecological Economics, ECOLEC-04558.

Ruiz-Agudelo, C.A., Bello, C., Londoño-Murcia, M. C., Alterio, H., Urbina-Cardona, J. N., Buitrago, A. Rodríguez Mahecha, J. V. (2011). Protocolo para la valoración económica de los Servicios Ecosistémicos en los Andes colombianos, a través del métodode transferencia de beneficios. Bogotá: Capital Natural Colombia. 
SEMARNAT. (2003). Introducción a los servicios ambientales. Secretaria de Medio Ambiente y Recursos Naturales (SEMARNAT) y Hombre naturaleza, México, D.F. México.

Tropical forest service flows: Improving our understanding of the biophysical dimension of ecosystem services. (2009). Forest Ecology and Management, (258), 1825-1829.

Vesterdal, L., Clarke, N., Sigurdsson, B. D. y Gundersen, P. (2013). Do tree species influence soil carbon stocks in temperate and boreal forests? Forest Ecology and Management, (309), 4-18.

Viglizzo, E. F., Paruelo, J. M., Laterra, P. y Jobbagy, E. G. (2012). Ecosystem service evaluation to support land-use policy. Agriculture, Ecosystems and Environment, (174), 78-84.

Villegas, C. I. (2012). Plan de valoración económica, ecológica y socio-cultural de bienes y servicios ambientales en la cuenca del Río Grande. Aproximación conceptual y metodológica. Seminario Internacional "Agronegocios y agroecología para el desarrollo. Medellín, Colombia: Universidad de Antioquia.

Vonada, R. (2010). Introducción a los servicios ambientales. Taller Regional: Compensación y Pago por Servicios Ambientales. La Ceiba, Honduras: Forest Trends

Volante, J.N., Alcaraz-Segura, D., Mosciaro, M.J., Viglizzo, E.F., Paruelo, J.M. (2012). Assessing the effect of land clearing on ecosystem services provision in north-western Argentina. Agriculture, Ecosystems and Environment 154, 1 